\title{
An fMRI study of imagined self-rotation
}

\author{
SARAH H. CREEM \\ University of Utah, Salt Lake City, Utah \\ TRACI HIRSCH DOWNS \\ University of Virginia, Charlottesville, Virginia \\ MARYJANE WRAGA \\ Smith College, Northampton, Massachusetts \\ GREGORY S. HARRINGTON \\ University of California, Davis, California \\ and \\ DENNIS R. PROFFITT and J. HUNTER DOWNS III \\ University of Virginia, Charlottesville, Virginia
}

\begin{abstract}
In the present study, functional magnetic resonance imaging was used to examine the neural mechanisms involved in the imagined spatial transformation of one's body. The task required subjects to update the position of one of four external objects from memory after they had performed an imagined self-rotation to a new position. Activation in the rotation condition was compared with that in a control condition in which subjects located the positions of objects without imagining a change in selfposition. The results indicated similar networks of activation to other egocentric transformation tasks involving decisions about body parts. The most significant area of activation was in the left posterior parietal cortex. Other regions of activation common among several of the subjects were secondary visual, premotor, and frontal lobe regions. These results are discussed relative to motor and visual imagery processes as well as to the distinctions between the present task and other imagined egocentric transformation tasks.
\end{abstract}

The ability to imagine what a scene looks like from another perspective develops in human beings quite early in life (Piaget \& Inhelder, 1948/1967). Many everyday tasks require this skill. For example, when giving a lecture, one might describe the layout of a slide from the audience's point of view. Such a task requires an imagined transformation of the egocentric reference frame, which specifies the up/down, front/back, and left/right axes of one's body. The ability to localize the objects on the slide from the audience's perspective requires the alignment of one's physical reference frame with the reference frame corresponding to the other view. In recent studies, researchers have investigated the neural mechanisms involved in egocentric imagery tasks requiring imagined actions directed toward objects (Decety et al., 1994; Grafton, Arbib, Fadiga, \& Rizzolatti, 1996) as well as implicit mental transformations of whole bodies and body parts to match pictures (Kosslyn, Digirolamo, Thompson, \& Alpert, 1998; Par-

This research was supported by NIMH Grant MH52640, NASA Grant NCC2925, and DARPA Grant 539689-52273. The authors thank Andrew Snyder for assistance in data collection. Correspondence concerning this article should be addressed to S. Creem, University of Utah, Department of Psychology, 380 S. 1530E. Rm. 502, Salt Lake City, UT 84112 (e-mail: sarah.creem @ psych.utah.edu). sons et al., 1995; Zacks, Rypma, Gabrieli, Tversky, \& Glover, 1999). However, no neuroimaging studies have examined imagined whole-body movement within the context of updating the positions of several external objects relative to oneself. In the present study, functional magnetic resonance imaging (fMRI) was used to identify the neural substrates involved in explicit imagined movement of the body to a new perspective. Specifically, we examined whether the evidence would support both visual-spatial and motor imagery processes that are seen in other egocentric imagery tasks.

Behavioral (e.g., Amorim \& Stucchi, 1997; Presson, 1982; Wraga, Creem, \& Proffitt, 2000) and neuroimaging (Bonda, Petrides, Frey, \& Evans, 1995; Kosslyn et al., 1998; Zacks et al., 1999) studies have suggested that egocentric transformation tasks are distinct from tasks that require the mental transformation of objects in several ways. In a cognitive task involving updating the positions of objects in space after imagined rotation, Wraga et al. (2000) found an advantage in both response latency and accuracy for viewer rotation compared with rotation of the objects themselves. Some neuroimaging studies suggest that motor areas are involved in egocentric but not object-relative transformations. In a PET study, Kosslyn et al. (1998) found evidence that motor processes play a role in the mental ro- 
tation of drawings of hands but not of cube figures. Several other neuroimaging studies have used hand judgment tasks to study the nature of egocentric transformations using PET (Bonda et al., 1995; Parsons et al., 1995). In general, these studies have characterized egocentric transformations with activity in the superior parietal lobule and both cortical and subcortical motor structures. Using fMRI, Zacks et al. (1999) suggested that there might be more left lateralization for egocentric transformations than for object-relative transformations.

In addition to their implications for motor imagery, imagined rotation tasks also introduce information about the mechanisms involved in visual imagery. There is evidence that visual processing mechanisms are active when visual imagery is performed (Cohen et al., 1996; Kosslyn et al., 1993: Kosslyn, Thompson, Kim, \& Alpert, 1995). Although primary visual cortex has been shown to be active in some visual imagery tasks (Kosslyn et al., 1999; Kosslyn et al., 1995), studies of mental rotation have indicated activity only in secondary visual areas (Cohen et al., 1996; Kosslyn et al., 1998).

In the present study, we examined the neural mechanisms involved in egocentric transformation, addressing several unique components. First, unlike most neuroimaging studies of mental rotation, our task did not have a visual stimulus present during scanning. In this way, we were able to assess the visual nature of imagined egocentric rotation without having a visual component to the stimulus. Second, our task involved explicit body movement to a new perspective and did not require the updating of bodypart positions. Instead, the imagined rotation was embedded within a task that explicitly required the updating of the positions of four external objects in the environment relative to oneself. This task allowed for an examination of "extrinsic" egocentric encoding of object location relative to the self (Buxbaum \& Coslett, 2000), as opposed to "intrinsic" spatial coding, which specifies the dynamic positions of body parts with respect to each other. Previous behavioral studies have shown that the spatial updating paradigm used in the present study yields extremely consistent performance. Compared with imagined rotation of objects, updating after imagined self-rotations is performed quickly and accurately, even when the rotation involves imagined movements against gravity (Creem, Wraga \& Proffitt, 2001; Wraga et al., 2000). Wraga et al. have suggested that people have a unique ability to imagine moving their bodies holistically, but that they cannot do the same with objects. Creem et al. also suggested that imagined self-movements may not follow the same constraints as imagined rotations of objects or of body parts, which have been suggested to require transformations through continuous points in space, analogous to those in the real world. Our goal was to assess the visual and motor mechanisms involved in imagined self-movement with the use of a well-established behavioral paradigm in fMRI. Our results indicated activation of a network including superior parietal, premotor, and secondary visual areas, mostly consistent with the findings of previous implicit body-part rotation tasks.

\section{METHOD}

\section{Subjects}

Twelve healthy right-handed volunteers ( 6 male, 6 female) aged 20-33 years (average age, 24 years) participated in the study. Handedness was assessed with a modified version of the Edinburgh handedness scale (Oldfield, 1971). All subjects gave written informed consent to the protocol as approved by the University of Virginia's human subjects committee.

\section{Procedure and Design}

Before beginning the task, the subjects learned that each of their four fingers represented a specific object. They learned to respond by pressing the button corresponding to the object that they were to name. None of the buttons corresponded spatially to the positions of the objects in the array. The subjects were given a practice session outside of the magnet on the day of testing in which they were taught the finger mapping of objects to buttons and performed an entire run of the task. The positions of the objects in the array were different in the practice session from those in the actual scanning session.

In the behavioral task, the subjects viewed a picture of a diamondshaped array of four objects (bed, hammer, teapot, car, as is shown in Figure 1A) presented in the viewer's frontal plane, and they memorized the positions of the objects. They were told to think of themselves as lying down in the middle of the array so that the objects were perceived as being in front, in back, to the left, and to the right of their bodies (see Figure 1B). After the subjects memorized the objects to a criterion of $100 \%$ correct, the visual display was removed and the rotation task was performed from memory. To assess memory, we required the subjects to close their eyes and to name the objects, given a position, randomly. If the subjects did not name the object correctly within $1 \mathrm{sec}$, they were instructed to study the objects again and were tested again in the same manner. On each trial, the subjects were told the degree to which they were to imagine rotating and a position in the array (i.e., " $90^{\circ}$, what is on the right?"). They imagined rotating clockwise (like a "log-roll") to the given amount and then responded with the name of the object that corresponded to the given position after the rotation. Reaction time (RT) and accuracy were recorded. The subjects were instructed to imagine themselves as being in their original positions at the beginning of each trial.

The rotation task consisted of three degrees of rotation $\left(90^{\circ}, 180^{\circ}\right.$, $270^{\circ}$ ), and the control task included only $0^{\circ}$ rotations. In effect, both tasks involved a spatial orientation decision (e.g., what is on the right?), but only the rotation condition required an imagined transformation.

In the scanner, a picture of the array (created with three dimensional [3-D] graphics software, ALICE) was initially presented through MR-compatible goggles (Resonance Technology, $30^{\circ} \mathrm{FOV}$ ). These goggles projected the image from an IBM laptop. The auditory stimulus was presented through MR-compatible stereo headphones that included approximately $30 \mathrm{~dB}$ of gradient noise cancellation. Auditory stimulus presentation was controlled by the experimental software (SuperLab, Cedrus, San Pedro, CA). In testing, the visual stimulus was removed, and the subjects were instructed to keep their eyes closed.

The subjects performed 12 epochs of trials, beginning with the control and alternating between the rotation and control tasks. There were 6 trials in each epoch for a total of 36 control $\left(0^{\circ}\right)$ trials and 36 rotation $\left(12\right.$ each of $90^{\circ}, 180^{\circ}$, and $\left.270^{\circ}\right)$ trials. The subjects initiated each subsequent trial with their response in the previous trial. Because the trials were self-initiated, epochs of trials varied in length, averaging about $20-30 \mathrm{sec}$ each. Since the $0^{\circ}$ trials were performed more quickly than the rotation trials, a 1-sec delay was added before the initiation of the auditory question in the control task trials. This allowed for equality in the number of volumes in each epoch. The average number of volumes for the control and rotation epochs was 7.53 (SD 1.0) and 7.68 (SD 1.36), respectively. RTs and errors were recorded using SuperLab. 

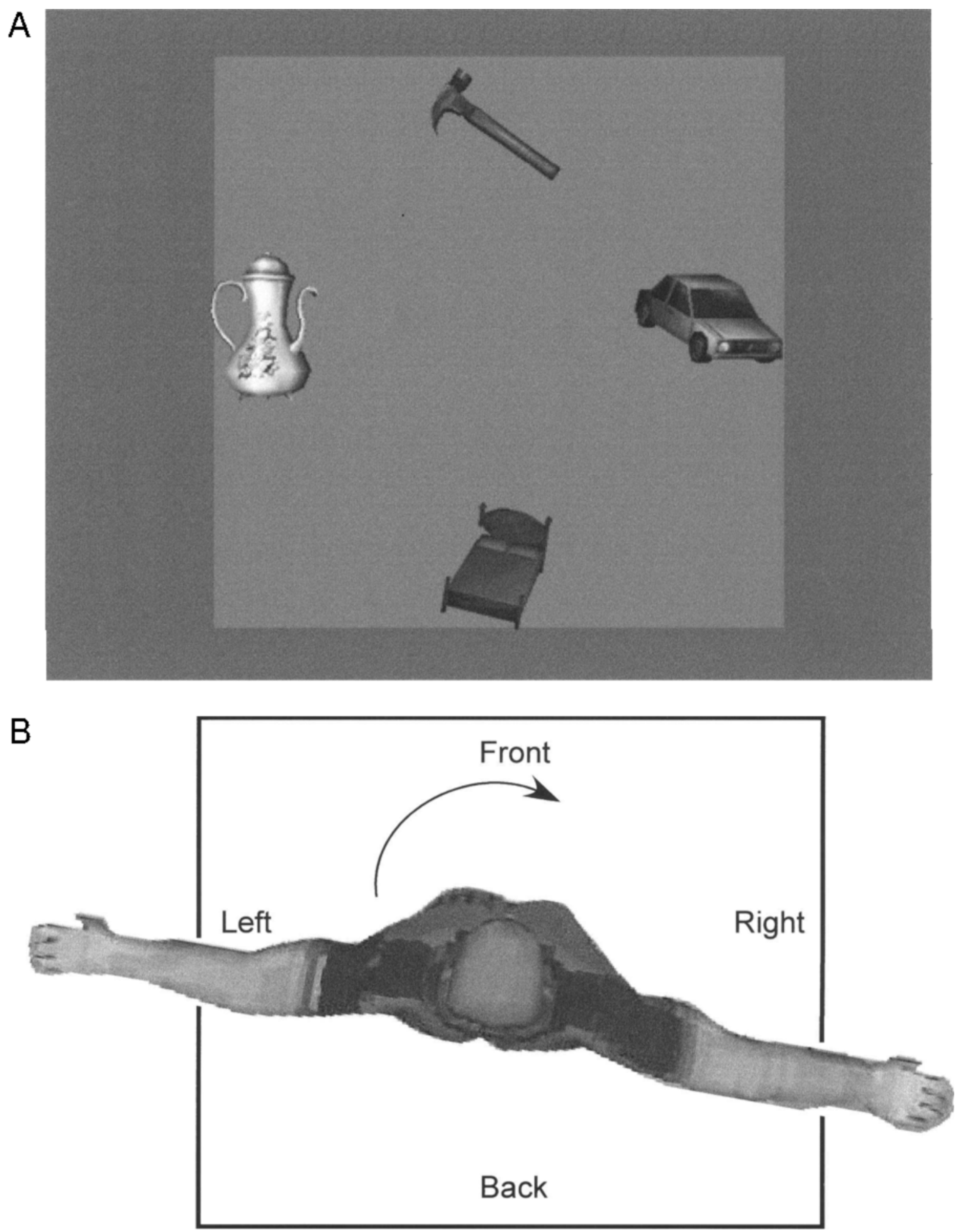

Figure 1. (A) Array of objects presented to the subject. (B) Schematic drawing of the imagined position of the subject relative to the array of objects.

\section{MRI Acquisition}

Each subject was fitted with a custom-made thermoplastic mask that minimized motion to less than $1 \mathrm{~mm}$. Once the subject was in the MRI scanner (1.5 Tesla Siemens Vision, Erlangen, Germany), a three-axis scout series was acquired for the positioning of the slices used for functional imaging. While positioning these slices, a 3-D highresolution T1-weighted image (MPRAGE, Mugler \& Brookeman, 1990) was acquired for anatomical localization of functional activa- 
Table 1

Clusters of Activation $>50 \mathbf{~ m m}^{3}$ for Averaged Thresholded Data in the Rotation Versus Control Task

\begin{tabular}{|c|c|c|c|c|c|c|}
\hline Area & $\begin{array}{l}\text { Brodmann } \\
\text { Area }\end{array}$ & $X$ & $Y$ & $Z$ & $\begin{array}{c}\text { Percentage of } \\
\text { Maximum Intensity }\end{array}$ & $\begin{array}{c}\text { Cluster } \\
\text { Size }\left(\mathrm{mm}^{3}\right) \\
\end{array}$ \\
\hline Left precuneus & 7 & -20 & -74 & 50 & .69 & 5,440 \\
\hline Right superior parietal lobule & 7 & 17 & -64 & 61 & .75 & 917 \\
\hline Left superior parietal lobule & 7 & -18 & 56 & 66 & .66 & 421 \\
\hline Left precentral gyrus & 6 & -43 & -3 & 38 & .43 & 285 \\
\hline Left postcentral gyrus & 7 & -5 & -53 & 65 & .39 & 229 \\
\hline Left superior frontal gyrus & 9 & -25 & 55 & 27 & .45 & 225 \\
\hline Left culmen & Cerebellum & -39 & -47 & -24 & .45 & 198 \\
\hline Left precentral gyrus & 6 & -25 & -13 & 67 & .32 & 186 \\
\hline Right declive & Cerebellum & 5 & -69 & -21 & .39 & 170 \\
\hline Left precuneus & 7 & -8 & -64 & 43 & .33 & 134 \\
\hline Right middle frontal gyrus & 11 & 30 & 42 & -14 & .27 & 116 \\
\hline Left cuneus & 19 & -3 & -93 & 26 & .27 & 102 \\
\hline Left inferior frontal gyrus & 9 & -54 & 7 & 33 & .33 & 90 \\
\hline Left middle frontal gyrus & 9 & -33 & 10 & 29 & .32 & 81 \\
\hline Left inferior frontal gyrus & 10 & -44 & 54 & 1 & .29 & 60 \\
\hline
\end{tabular}

Note-Intensity is the parameter $\alpha$ in the least-squares fit of each voxel time series $x(t)=\alpha * r(t)+a+b * t+$ noise where $r(t)$ is the known reference waveform representing the expected time course, $\alpha$ is the amplitude of the activation, $a$ is the mean signal level, and $b$ is the linear time drift.

tion sites and was partitioned into 100 contiguous 1.5 -mm sagittal slices $1 \times 1 \mathrm{~mm}$ in plane resolution $(256 \times 256$ matrix $)$. Next, each functional condition was imaged with the use of a maximum of 114 volumes ${ }^{1}$ of $25-27$ contiguous (4-mm) axial slices (the number of slices was varied in order to avoid artifacts in the frontal sinus). The functional acquisition was continuous across epochs. The sequence used for functional images was a gradient echo-planar sequence $\left(\mathrm{TR}=3.05 \mathrm{sec}, \mathrm{TE}=48 \mathrm{msec}, \mathrm{FOV}=335 \mathrm{~mm}\right.$, flip angle $=90^{\circ}$, readout bandwidth $=2080 \mathrm{~Hz} /$ pixel $)^{2}$ and the resulting images were reconstructed into a $128 \times 128$ voxel matrix with an in-plane voxel size of $2.62 \times 2.62 \mathrm{~mm}$. Total imaging time was approximately $25 \mathrm{~min}$.

\section{Imaging Analysis}

Image analysis was performed off line using the AFNI software (Cox, 1996). All functional images were motion corrected with the 3-D algorithm (Cox \& Jesmanowicz, 1999) within AFNI. The anatomical images were realigned and normalized to the standard anatomical space defined by Talairach and Tournoux (1988). In order to examine individual data, significantly activated functional areas for each subject were determined by using the correlation method that was first described by Bandettini (1993), and the resulting correlation coefficient images were thresholded to a significance level of $p<.0005$. (The program AlphaSim, part of the AFNI package, was used to estimate the necessary cluster size to achieve a significance level of .05 with an individual voxel threshold of $p<$ .0005.) Correlation coefficient images were then transformed to the standard Talairach space by using the transform derived from the anatomical data. These images were then blurred with the use of a Gaussian filter with a full-width half maximum (FWHM) of $6 \mathrm{~mm}$ to compensate for residual anatomical differences after normalization. For an initial group analysis of trends, functional intensities of the voxels that passed the individual threshold for each of the 12 subjects were averaged. ${ }^{3}$ Talairach coordinates and probable locations were then determined for all of the averaged significantly activated points by using the Talairach Daemon (Lancaster, Summerln, Rainey, Freitas, \& Fox, 1997). The clusters are presented in Table 1. In a second group analysis, ${ }^{4}$ each individual statistical map (no threshold) was transformed to Talairach space and smoothed with a 6-mm FWHM Gaussian filter. Group averages of activations and deactivations were created by calculating the mean of the correlation

Table 2

Clusters of Activation and Deactivation in the Average Correlation Map

\begin{tabular}{lrrrrrr}
\hline \multicolumn{1}{c}{ Area } & $\begin{array}{c}\text { Brodmann } \\
\text { Area }\end{array}$ & \multicolumn{1}{c}{$X$} & $Y$ & $Z$ & $t$ score & $\begin{array}{c}\text { Cluster } \\
\text { Size }\left(\mathrm{mm}^{3}\right)\end{array}$ \\
\hline Left precuneus & 7 & -12 & -74 & 52 & 4.63 & 894 \\
Right precuneus & 7 & 16 & -64 & 47 & 4.58 & 337 \\
& \multicolumn{7}{c}{ Deactivations } \\
Right anterior cingulate & 24 & 5 & 30 & 1 & 4.14 & 26,254 \\
Right superior frontal gyrus & 8 & 7 & 43 & 47 & 4.03 & 3,273 \\
Left cingulate gyrus & 31 & -3 & -40 & 31 & 3.99 & 1,774 \\
Left inferior frontal gyrus & 47 & -32 & 25 & -13 & 4.01 & 1,335 \\
Left superior frontal gyrus & 8 & -14 & 37 & 50 & 3.90 & 889 \\
Right middle frontal gyrus & 6 & 44 & 11 & 46 & 4.45 & 860 \\
Right inferior frontal gyrus & 45 & 52 & 20 & 11 & 4.36 & 782 \\
Right supramarginal gyrus & 40 & 55 & -37 & 34 & 4.47 & 490 \\
Left inferior parietal lobule & 40 & -56 & -29 & 25 & 4.31 & 451 \\
Right superior frontal gyrus & 9 & 17 & 57 & 38 & 3.98 & 337 \\
Right precuneus & 7 & 23 & -45 & 52 & 3.95 & 330 \\
\hline
\end{tabular}

Note-Threshold at $t=3.5, p<.005$, cluster $>300 \mathrm{~mm}^{3}$. 
values for each voxel and the corresponding $t$ statistic of the mean. A functional map was created by applying a threshold of $p<.005$ and a cluster size $>300 \mathrm{~mm}^{3}$.

\section{Results}

Behavioral results. Figure $2 \mathrm{~A}$ presents the RT data for the fMRI viewer task in which the subjects imagined rotations of $0^{\circ}, 90^{\circ}, 180^{\circ}$, and $270^{\circ}$ and spatially updated the positions of objects. The results were similar to the behavioral results for the viewer task reported in Wraga et al. (2000) in which subjects stood inside (Experiment 2) an array of four objects and imagined rotating themselves in a manner similar to that in the present experiment. A repeated-measures analysis of variance (ANOVA) was performed on the data from 6 subjects in the present study with degree of rotation as the within-subjects variable. 5 The analysis indicated a significant effect of degree of rotation $[F(3,15)=4.17, p<.05]$. Planned repeated contrasts revealed that response latency at $90^{\circ}$ was greater than that at $0^{\circ}[F(1,5)=10.94, p<.021]$, but there were no differences between the greater degrees of rotation $\left(90^{\circ} \mathrm{vs}\right.$. $180^{\circ}, p=.26 ; 180^{\circ}$ vs. $\left.270^{\circ}, p=.24\right)$. Wraga et al. (2000) distinguished between updating after imagined viewer and object rotations, finding notably different RT and accuracy functions. Their results indicated a large RT advantage in the viewer task compared with that in the array task. Furthermore, RT increased up to the $270^{\circ}$ rotation in the array task, but peaked at either $90^{\circ}$ or $180^{\circ}$ in the viewer task. The consistency of the present results with those in the viewer task in Wraga et al. leads us to conclude that the subjects were imagining the movement of their own bodies and not of the objects. The fast RT at $270^{\circ}$ in the viewer task is noteworthy and consistent with previous results. It may be that subjects imagine rotating in the opposite direction or that they are able to instantly transport themselves to a new viewpoint rather than rotating through all the points in space, as has been suggested by objectrotation studies (e.g., Shepard \& Metzler, 1971).

Figure 2B presents the accuracy data for the same 6 subjects. In all, percent of correct responses was high. A repeated-measures ANOVA with degree of rotation as the within-subjects variable revealed an effect of degree of rotation $[F(3,15)=5.57, p<.01]$. Planned simple contrasts indicated that the accuracy for the $180^{\circ}$ rotation was lower than that for $0^{\circ}[F(1,5)=33.35, p<.01]$, but no other degrees of rotation differed from $0^{\circ}\left(90^{\circ}, p=.52 ; 270^{\circ}, p=\right.$ .18). Post hoc tests indicated that accuracy at $180^{\circ}$ differed from that at $90^{\circ}[F(1,5)=11.95, p<.02]$ but did not differ from $270^{\circ}[F(1,5)=2.29, p<.19]$. The high level of accuracy is consistent with the findings for the viewer task in Wraga et al. (2000).

Because the number of subjects was smaller than those we have tested in previous behavioral studies, and because we were unable to analyze the data from 6 subjects in the present study, we conducted an additional behavioral study using a cot outside of the magnet, with exactly the same paradigm. The subjects performed alternating blocked trials of the control and rotation tasks. The RT and accuracy performance of 15 subjects ( 7 female, 8 male) was analyzed. ${ }^{6}$ Figure 3 illustrates that the RT and accuracy data for the follow-up study closely resembled the data obtained from the 6 subjects in the scanner. A repeatedmeasures ANOVA with degree of rotation as the withinsubjects variable indicated a significant effect of degree $[F(3,42)=17.70, p<.001]$. Planned repeated contrasts indicated that RT was significantly greater at $90^{\circ}$ than at $0^{\circ}$
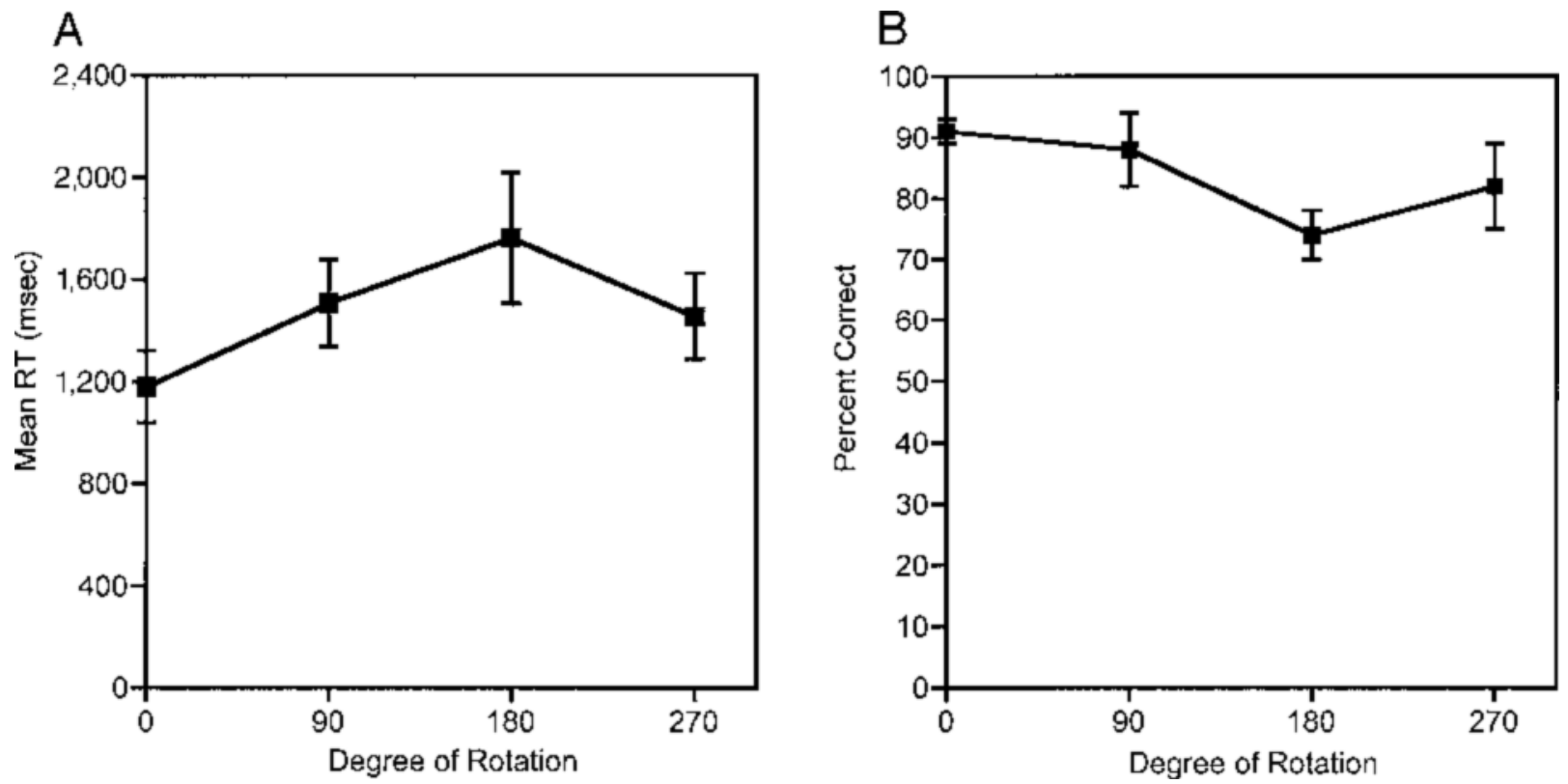

Figure 2. (A) Mean reaction time $( \pm 1 S E$ ) as a function of degree of rotation for the viewer task performed in the scanner. (B) Mean percent of correct responses $( \pm 1 S E)$ as a function of degree of rotation for the viewer task performed in the scanner. 

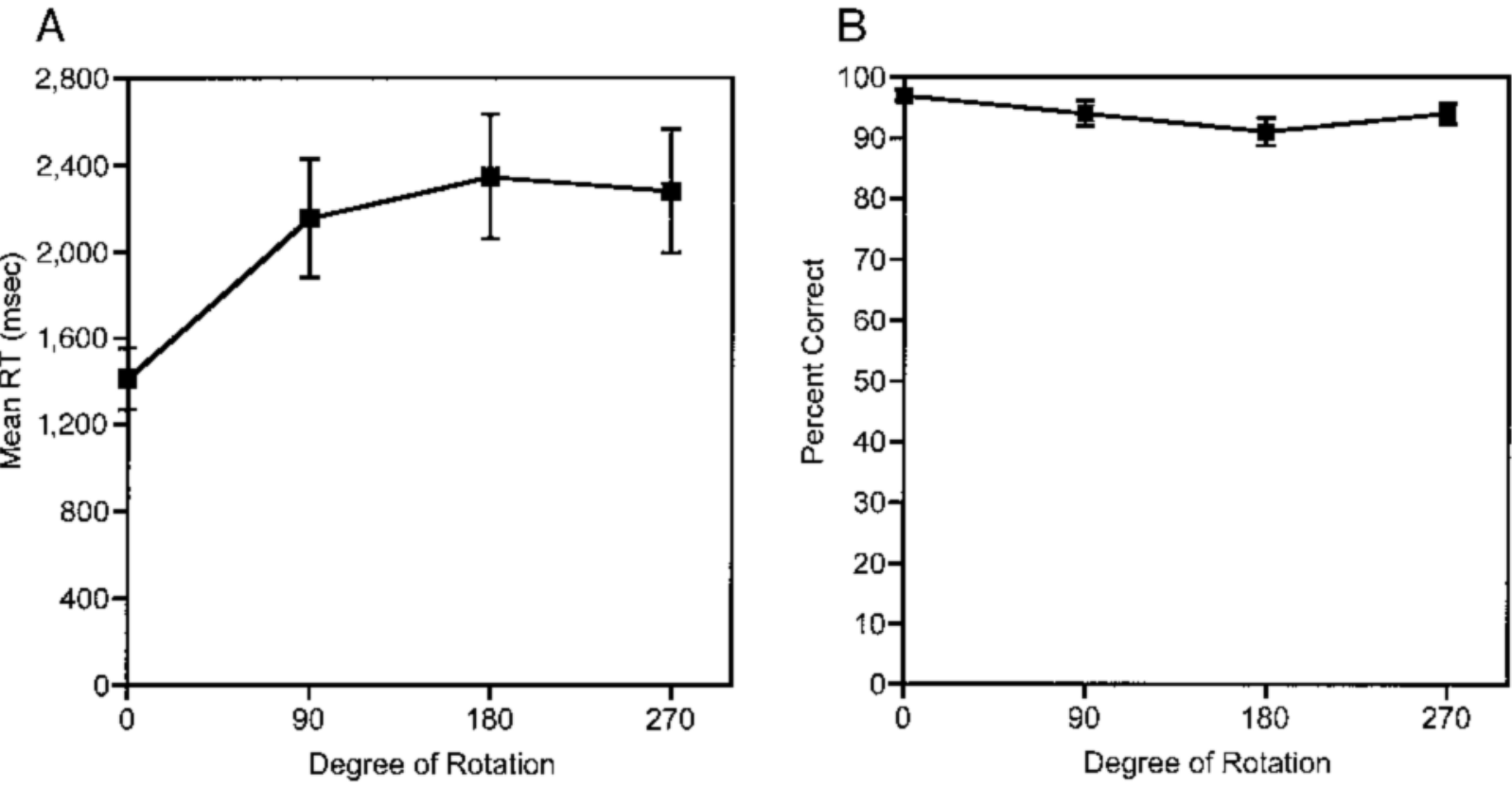

Figure 3. (A) Mean reaction time $( \pm 1 S E)$ as a function of degree of rotation for the follow-up behavioral study of viewer rotation. (B) Mean percent of correct responses $( \pm 1 S E)$ as a function of degree of rotation for the follow-up behavioral study of viewer rotation.

$[F(1,14)=25.0, p<.001]$ but that there was no difference between greater degrees of rotation $\left(90^{\circ}\right.$ vs. $180^{\circ}, p=.17$; $180^{\circ}$ vs. $270^{\circ}, p=.50$ ). For the accuracy measurement, the subjects demonstrated as high a level of performance as in the scanner, showing a slight drop in performance at $180^{\circ}$; however, the ANOVA revealed no differences as a function of rotation $[F(3,42)=2.17, p<.11]$ (see Figures $3 \mathrm{~A}$ and $3 \mathrm{~B}$ ). The results of this follow-up behavioral study largely support the findings of the initial study during scanning.

Imaging results. The individual and group average analyses indicated a network of secondary visual, parietal, and frontal areas (see Table 1). The second group $t$ test indicated the predominance of significant posterior parietal activity, with strong lateralization to the left hemisphere (see Table 2). ${ }^{7}$ Table 2 also presents statistically significant decreases of activation in the rotation task, as compared with the control task. The differences between the significant group correlation map (activations in Table 2) and the average map based on individual thresholded data (Table 1) may be attributed to the variability seen between some subjects. We present the group average based on the individual thresholded data to illustrate the multiple trends of stronger activation in the rotation task, as compared with the control task. However, the fact that not all the subjects showed activation in all of these areas is indicated in the results and will be discussed.

The principal question in the present study was whether an imagined self-rotation task involving the entire body would recruit brain activity similar to other egocentric body-part decision tasks. An area strongly associated with egocentric mental rotation tasks has been the superior parietal lobule. Figure 4 illustrates significant activity in the left precuneus (BA 7), the most robust area of activation in the present study (10/12 subjects). Statistically significant right superior parietal lobule/precuneus activity was found as well (8/12 subjects). For visual areas, we found trends of activation in the left cuneus (BA 19, 5/12 subjects). The subjects also showed activation in Brodmann Area 6, premotor area, both ventral (5/12 subjects) and dorsal (6/12 subjects) regions of the left precentral gyrus. In addition to these areas, several other regions associated with motor processing were apparent in some subjects (see Table 1). Subcortically, we found activation in bilateral regions of the cerebellum. This activation is consistent with the notion that the subjects may have imagined movement of their own bodies to solve the task. Furthermore, several frontal lobe regions showed increased activation for some subjects-namely, the left superior, middle, and inferior frontal gyri (BAs 9/10). These prefrontal areas have been associated with other imagined rotation tasks (Cohen et al., 1996; Kosslyn et al., 1998) as well as with other tasks involving the maintenance and manipulation of information in memory (Fletcher \& Henson, 2001).

Recent efforts have been made to systematically describe and interpret deactivations present in neuroimaging tasks (Raichle et al., 2001; Shulman et al., 1997). In the present study, we found several large clusters of decreased activation when we compared the rotation task with the control task (see Table 2). Notably, these activations in medial frontal regions (anterior cingulate, superior frontal gyrus, cingulate), dorsolateral frontal cortex, and inferior parietal cortex are similar to those reported by Shulman et al. in a comprehensive analysis of active 


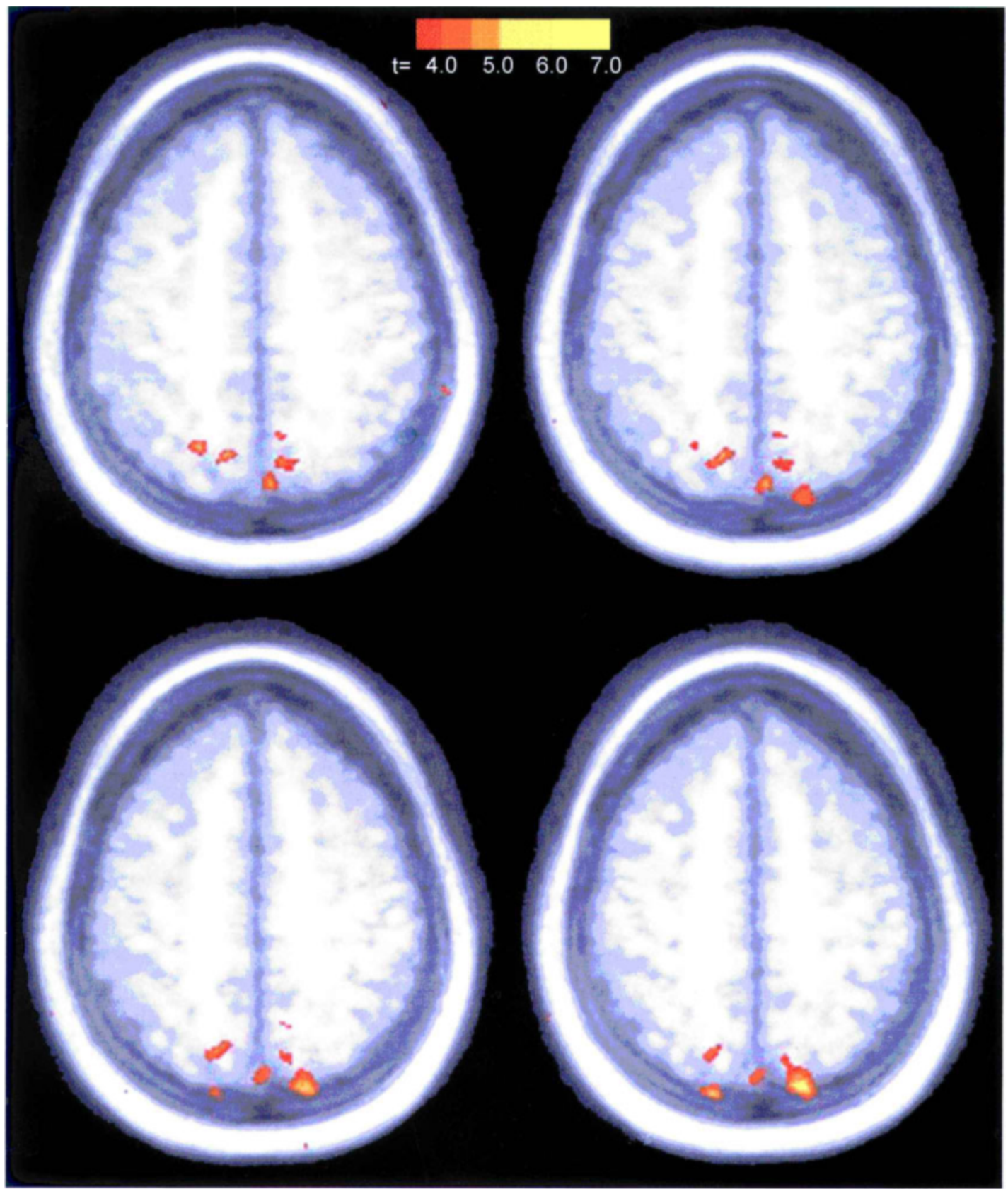

Figure 4. (A) Left and right precuneus/superior parietal lobule, BA 7 ( $z=48-51)$. Images are presented in radiological conventions $($ left $=$ right, right $=$ left $)$.

versus passive states in nine visual processing PET studies. There are several possible interepretations for the large clusters of decreased activation. Drevets and Raichle (1998) have suggested that ventromedial frontal cortex may be inhibited during difficult cognitive tasks. Shulman et al. (1997) also suggested that the deactivations found in their meta-analysis may have been a result of unconstrained verbal thought, or the monitoring of one's external environment, one's body image, or one's emotional state. These possible interpretations are based on a low- level passive viewing task. Unlike these tasks, our control task involved an egocentric memory task in which subjects were required to recall the object in a specific location. An alternative explanation is increased activity caused by the passive task memory processes themselves.

\section{GENERAL DISCUSSION}

We investigated whether a task of imagined self-rotation relative to an external array of objects would recruit neural 
areas subserving visuospatial and motor processing similar to those found in other egocentric imagery tasks (i.e., right-left hand decision). The results indicate that our imagined self-rotation task recruited areas similar to those found in implicit hand-rotation tasks (e.g., Kosslyn et al., 1998; Parsons et al., 1995), with the exception that those studies found primary motor cortex involvement (Ganis, Keenan, Kosslyn, \& Pascual-Leone, 2000; Kosslyn et al., 1998). We found that secondary visual, parietal, and premotor areas showed more significant activation in the rotation task, as compared with the control task. Our design allowed us to specifically test egocentric perspective transformations. Both the rotation and the control tasks required an egocentric decision about the spatial positions of objects, but in addition, the rotation task required an imagined transformation of the egocentric reference frame. The results are discussed in the framework of the involvement of both visual-spatial and motor imagery processes in imagined self-rotation.

\section{Imagined Rotation and Visual Processing}

Within cognitive research, it is well established that imagining the rotation of an object recruits visual perceptual mechanisms (see Shepard \& Cooper, 1982; Shepard $\&$ Metzler, 1971). Recent neuroimaging studies of mental rotation as well support the notion of shared neural systems for visual perception and visual imagery using pictures of both objects and hands (Alivisatos \& Petrides, 1997; Bonda et al., 1995; Cohen et al., 1996; Kosslyn et al., 1998). Compared with various visual baseline conditions, the rotation tasks consistently activated secondary visual areas (BAs 18 and 19). For example, Kosslyn et al. (1998) asked subjects to discriminate whether drawings of two cube figures (or hands) were the same or different. They found that the cube-rotation task led to bilateral activation of BA 19. For hands, they found activation in BA 19 in the left hemisphere and in primary visual cortex (BA 17) at the midline. Cohen et al. (1996) did not find primary visual cortex activation in a cube-rotation task, but did find activation in cortical area V5, which is known to respond to motion of stimuli (Tootell et al., 1995).

Our results extend these findings to mental transformations that are performed without the presence of any visual stimulus. Imagining one's own transformation of perspective from memory led to increased activity in secondary visual areas. We found the secondary visual areas active in 9 out of 12 subjects. We did not find activation in primary visual cortex. It could be that our high-level imagery control factored out the primary visual cortex activity that has been seen in image-generation tasks (Kosslyn et al., 1995).

\section{Imagined Rotation and Spatial Processing}

Visual-spatial processing is associated with neural activity in several distinct areas. The finding of bilateral posterior parietal activation, with extensive activation lateralized to the left cerebral hemisphere, is consistent with numerous studies of mental rotation (Alivisatos \& Petrides, 1997; Kosslyn et al., 1998; Richteret al., 2000; Tagaris et al., 1996,
1997) and is consistent with the results of other visuospatial tasks involving the egocentric reference frame and the encoding of spatial relations (e.g., Aguirre \& D'Esposito, 1997). To assess the similarity of the posterior parietal activation in the present study with that of previous findings, a region of interest analysis was performed. We identified a region encompassing the border between the left precuneus and superior parietal lobule found in Kosslyn et al. (1998), Parsons et al. (1995), ${ }^{8}$ and Alivisatos and Petrides (1997) $(x=0,-21, y=-67,-84, z=42,52)$. We found an activation 9 cluster of $445 \mathrm{~mm}^{3}$ in that region.

Research with neuropsychological patients also supports the role of the superior parietal lobe in egocentric spatial tasks. Patients with posterior parietal lesions exhibit disturbances of spatial body knowledge (De Renzi, 1982), egocentric visually guided actions (Jeannerod, Decety, \& Michel, 1994), and spatial attention (Heilman, Watson, \& Valenstein, 1993). The superior parietal lobe is defined as the endpoint of the dorsal visual processing stream, which transforms visual information using an egocentric coordinate system (Milner \& Goodale, 1995). Our findings are consistent with this role in egocentric encoding of space. The posterior parietal area, with direct projections to premotor areas, has been consistently associated with egocentric spatial processing for planning and executing actions.

Laterality is also important to consider. The presence of left hemisphere lateralization supports Zacks et al. (1999), who found activity in the left parietal-temporaloccipital junction in a task that required a left-right judgment about a human figure from the figure's perspective. Kosslyn et al. (1998) also found left hemisphere activity for their hand-rotation task, but bilateral activation for the cube-rotation task. Other implicit hand-rotation tasks have consistently found bilateral superior parietal activation (Bonda et al., 1995; Parsons et al., 1995). In contrast, other egocentric and allocentric spatial-judgment tasks, not involving a spatial transformation, have indicated primarily right hemisphere parietal activation (Galati et al., 2000; Vallar et al., 1999). Together, the present and past rotation studies support the notion that the left superior parietal region is necessary for egocentric transformation tasks and that some tasks recruit the right hemisphere as well.

\section{Imagined Rotation and Motor Processing}

Evidence for motor processing was also found. The results indicated left hemisphere activation in BA 6, the premotor cortex (dorsal, found in 6/12 subjects; ventral, found in 5/12 subjects). BA 6 is known to be involved in preparation for movement and motor planning. Furthermore, it has direct connections with regions of the posterior parietal lobe (He, Dumm, \& Strick, 1995). In addition, we found some activation in Prefrontal Areas 9, 10, and 11 , and in bilateral regions of the cerebellum. These areas have been associated with spatial working memory. Specifically, areas within the dorsolateral prefrontal cortex in the monkey receive input from the posterior parietal cortex and have been shown to be essential for guiding choices in spatial memory tasks (Passingham, 1993). Re- 
cently, several lines of evidence have indicated that motor mechanisms play an important role in mental rotation. There are two relevant questions about the nature and extent of motor representations involved in mental rotation: (1) the extent to which motor processing is involved in all imagined rotations and (2) whether primary motor cortex is involved in motor imagery. With regard to the first question, it has not been clear in the literature whether motor processing is associated with all imagined rotations, or only with the rotation of body parts. Kosslyn et al. (1998) found motor activity (M1 and premotor cortex) in a handrotation task, but did not find any frontal motor areas associated with a cube-rotation task. Most other studies involving the implicit rotation of hands have found premotor activity (Bonda et al., 1995; Parsons et al., 1995). In Cohen et al.'s (1996) cube-rotation task using fMRI, one half of the subjects also showed premotor activation. Richter et al. (2000) found bilateral premotor and supplementary motor area activation in a cube-rotation task. Research from behavioral studies, as well, indicates the involvement of motor processing in classic mental rotation tasks using Shepard cube figures (Wexler, Kosslyn, \& Berthoz, 1998; Wohlschläger \& Wohlschläger, 1998). One interpretation of the findings of motor involvement in mental rotation of objects is that observers use a motor strategy of imagining a hand rotating the object. Using PET, Kosslyn, Thompson, Wraga, and Alpert (2001) compared activation for mental rotation of cubes given an internal hand-rotation strategy or an externalmotor-rotation strategy. They found that the internal strategy, to imagine rotating objects with the dominant hand, showed premotor and primary motor activation that was not as apparent with the external strategy (imagined rotation of the object as if it were driven by an external motor). Our present study extends the evidence for the recruitment of motor areas to whole-body perspective transformations in some, but not all, subjects. Individual differences may be a result of differences in the strategies used to solve the viewer rotation task. Some might have imagined "rolling" through space; others might have been able to change their perspective without actually performing the rotation.

Some debate exists over whether primary motor cortex (M1) is associated with imagined movement. Although several studies have not found this activation in imagined motor tasks (Decety et al., 1994; Parsons et al., 1995; Rao et al., 1995; Stephan et al., 1995), other recent studies present evidence for the involvement of M1 during motor imagery (e.g., Ganis et al., 2000; Porro et al., 1996; Kosslyn et al., 1998; Wraga et al., 2000). Recently, Ganis et al. (2000) tested the hypothesis that primary motor cortex is needed for the mental rotation of hands and feet by delivering singlepulse transcranial magnetic stimulation (TMS) to the hand area of the primary motor cortex. They concluded that the left primary motor cortex is involved in the mental rotation of hands and feet, but that it is stimulus specific, since stimulation applied to the hand area produced more of a decrement in performance for hands than for feet. In all, there are mixed results about the involvement of primary motor cortex in imagined motor tasks, which could be a result of differing stimuli, paradigms, or strategies.

\section{Whole-Body Perspective Versus Body-Part Transformation}

The present task can be distinguished from previous egocentric rotation tasks by the fact that it requires rotation of one's whole-bodyperspective rather than requiring rotation of a specific body part. This distinction may be one between extrinsic egocentric encoding of object location relative to the self (Buxbaum \& Coslett, 2000) and intrinsic spatial coding, specifying the dynamic positions of body parts with respect to each other (Vindras \& Viviani, 1998). Parsons $(1987,1994)$ illustrated that imagined rotation of body parts adheres to physical constraints of body movement. Latency to make a handedness judgment about a rotated body part is a function of the awkwardness of the implicit physical movement of the body part. In contrast, by using a task of imagined whole body rotations, Creem et al. (2001) found that rotations about axes that defied the law of gravity could be performed as easily as physically possible ones.

Taken together, the hand-rotation and body-rotation tasks may suggest a fundamental difference concerning adherence to physical motor constraints for intrinsic versus extrinsic egocentric decision tasks. Although the subjects in our study were instructed to rotate to a new position, they were able to imagine a perspective transformation, seemingly unlimited by physical constraints. As indicated by the relatively flat slope that we found for response latencies above $0^{\circ}$, the subjects seemed to be able to imagine themselves transported to a new perspective without their passing through all of the points in space (see also Wraga et al., 2000). In contrast, in hand- and foot-rotation tasks (Parsons, 1987, 1994; Parsons et al., 1995), it has been shown that observers solve the task by imagining their own body part fitting into the picture, a decision that apparently is constrained by biomechanical properties of the body. In these studies, the time it took to determine the handedness of a figure was a function of the degree of rotation from the figure's canonical orientation. Neurally, the present study found that body perspective transformations recruited some frontal lobe motor processing areas, but not in all subjects, and did not involve primary motor cortex. Thus, we might expect M1 and extensive premotor activity to be involved in motor imagery associated with body-part rotation (as seen in Ganis et al., 2000; Kosslyn et al., 1998) but not whole-body perspective rotation, because of the nature of the physical constraints of the former imagined task. Updating from a new perspective may be performed in multiple ways. If subjects were to use a spatial strategy that does not involve processes physically analogous to moving in space, we might expect to see a predominance of superior parietal activity tied to the egocentric perspective-taking task and to a lesser extent, neural structures tied to motor activity. 


\section{CONCLUSIONS}

In the present experiment, we examined the neural mechanisms involved in the mental transformation of the egocentric reference frame relative to an external array of objects. Our task was unique because it involved an extrinsic egocentric decision (relative to external objects) and required performance without vision. We found that activated regions of secondary visual areas, posterior parietal lobe, and areas of the frontal lobe were similar to those found in other egocentric handedness decision tasks. Most significantly, we found robust posterior parietal activity in the left hemisphere that may be attributed to egocentric transformations. This study provides a basis for understanding the neural processes involved in spatial transformations of one's own body. It suggests that extrinsic egocentric tasks share similar neural mechanisms to intrinsic egocentric tasks, but it is possible that the nature of the physical constraints of the transformation may differ. Future neuroimaging research should aim to directly compare intrinsic and extrinsic egocentric decision tasks as well as the differences in the strategies used to solve the task.

\section{REFERENCES}

Aguirre, G. K., \& D'Esposito, M. (1997). Environmental knowledge is subserved by separable dorsal/ventral neural areas. Journal of Neuroscience, 17, 2512-2518.

Alivisatos, B., \& Petrides, M. (1997). Functional activation of the human brain during mental rotation. Neuropsychologia, 35, 111-118.

Amorim, M.-A., \& STUcchi, N. (1997).Viewer- and object-centered mental explorations of an imagined environment are not equivalent. Cognitive Brain Research, 5, 229-239.

BANDETTINI, P. A. (1993). Processing strategies for time-course data sets in functional MRI of the human brain. Magnetic Resonance in Medicine, 30, 161-173.

Bonda, E., Petrides, M., Frey, S., \& Evans, A. (1995). Neural correlates of mental transformations of the body-in-space. Proceedings of the National Academy of Sciences, 92, 11180-11184.

Buxbaum, L. J., \& Coslett, H. B. (2000). Spatio-motor aspects of action. In B. Rapp (Ed.), The handbook of cognitive neuropsychology (pp. 543-563). Philadelphia: Psychology Press.

Cohen, M. S., Kosslyn, S. M., Breiter, H. C., DiGirolamo, G. J., Thompson, W. L., Anderson, A. K., Bookheimer, S. Y., Rosen, B. R., \& Belliveau, J. W. (1996). Changes in cortical activity during mental rotation: A mapping study using functional MRI. Brain, 119, 89-100.

Cox, R. W. (1996). AFNI: Software for analysis and visualization of functional magnetic resonance neuroimages. Computers \& Biomedical Research, 29, 162-173.

Cox, R. W., \& Jesmanowicz, A. (1999). Real-time 3D image registration for functional MRI. Magnetic Resonance in Medicine, 42, 10141018.

Creem, S. H., Wraga, M., \& Proffitt, D. R. (2001). Imagining physically impossible transformations: Geometry is more important than gravity. Cognition, 81, 41-64.

Decety, J., Perani, D., Jeannerod, M., Bettinardi, V., Tadary, B., Woods, R, Mazziotta, J. C., \& FAzIo, F. (1994). Mapping motor representations with positron emission tomography. Nature, 371, 600-602.

De RenZi, E. (1982). Disorders of space exploration and cognition. Chichester, U.K.: Wiley.

Drevets, W. C., \& Raichle, M. E. (1998). Reciprocal suppression of regional cerebral blood flow during emotional versus higher cognitive processes: Implications for interactions between emotion and cognition. Cognition \& Emotion, 12, 353-385.
Fletcher, P. C., \& Henson, R. N. A. (2001). Frontal lobes and human memory: Insights from functional neuroimaging. Brain, 124, 849881.

Galati, G., Lobel, E., Vallar, G., Berthoz, A., Pizzamiglio, L., \& LE BiHAN, D. (2000). The neural basis of egocentric and allocentric coding of space in humans: A functional magnetic resonance study. Experimental Brain Research, 133, 156-164.

Ganis, G., Keenan, J. P., Kossly n, S. M., \& Pascual-Leone, A. (2000). Transcranial magnetic stimulation of primary motor cortex affects mental rotation. Cerebral Cortex, 10, 175-180.

Grafton, S. T., Arbib, M. A., Fadiga, L., \& Rizzolatti, G. (1996). Localization of grasp representation in humans by positron emission tomography. Experimental Brain Research, 112, 103-111.

He, S. Q., Dumm, R. P., \& Strick, P. L. (1995). Topographic organization of corticospinal projections from the frontal lobe: Motor areas on the medial surface of the hemisphere. Journal of Neuroscience, 15, 3284-3306.

Heilman, K. M., Watson, R. T., \& Valenstein, E. (1993). Neglect and related disorders. In K. M. Heilman \& E. Valenstein (Eds.), Clinical neuropsychology (pp. 279-336). Oxford: Oxford University Press.

JeAnnerod, M., Decety, J., \& Michel, F. (1994). Impairment of grasping movements following a bilateral posterior parietal lesion. Neuropsychologia, 32, 369-380.

Kosslyn, S. M., Alpert, N. M., Thompson, W. L., Malijkovic, V., Weise, S. B., Chabris, C. F., Hamilton, S. E., Rauch, S. L., \& BuoNANNO, F. S. (1993). Visual mental imagery activates topographically organized visual cortex: PET investigations. Journal of Cognitive Neuroscience, 5, 263-287.

Kosslyn, S. M., Digirolamo, G. J., Thompson, W. L., \& Alpert, N. M. (1998). Mental rotation of objects versus hands: Neural mechanisms revealed by positron emission tomography. Psychophysiology, 35, 151-161.

Kosslyn, S. M., Pascual-Leone, A., Felician, O., Camposano, S., Keenan, J. P., Thompson, W. L., Ganis, G., Sukel, K. E., \& Alpert, N. M. (1999). The role of Area 17 in visual imagery: Convergent evidence from PET and rTMS. Science, 284, 167-170.

Kosslyn, S. M., Thompson, W. L., Kim, I. J., \& Alpert, N. M. (1995). Topographical representations of mental images in primary visual cortex. Nature, 378, 496-498.

Kosslyn, S. M., Thompson, W. L., Wraga, M., \& Alpert, N. M. (2001). Imagining rotation by endogenous versus exogenous forces: Distinct neural mechanisms. NeuroReport, 12, 2519-2525.

Lancaster, J. L., Summerln, J. L., Rainey, L. Freitas, C. S., \& Fox, P. T. (1997). The Talairach Daemon, a database server for Talairach Atlas Labels. NeuroImage, 5, S633.

Milner, A. D., \& Goodale, M. A. (1995). The visual brain in action. Oxford: Oxford University Press.

Mugler, J. P., III, \& Brookeman, J. R. (1990). Three-dimensional magnetization-prepared rapid gradient-echo imaging (3D MP RAGE). Magnetic Resonance in Medicine, 15, 152-157.

OldFIELD, R. C. (1971). The assessment and analysis of handedness: The Edinburgh Inventory. Neuropsychologia, 9, 97-113.

PARSONS, L. M. (1987). Imagined spatial transformations of one's hands and feet. Cognitive Psychology, 19, 178-241.

PARsons, L. M. (1994). Temporal and kinematic properties of motor behavior reflected in mentally simulated action. Journal of Experimental Psychology: Human Perception \& Performance, 20, 709-730.

Parsons, L. M., Fox, P. T., Downs, J. H., Glass, T., Hirsch, T. B., Martin, C. G., Jerabek, P. A., \& Lancaster, J. L. (1995). Use of implicit motor imagery for visual shape discrimination as revealed by PET. Nature, 375, 54-58.

Passingham, R. E. (1993). The frontal lobes and voluntary action. Oxford: Oxford University Press.

Piaget, J., \& INHelder, B. (1967). The child's conception of space. (F. J. Langdon \& J. L. Lunzer, Trans.) New York: Norton. (Original work published 1948)

Porro, C. A., Francescato, M. P., Cettolo, V., Diamond, M. E., Baraldi, P., Zuiani, C., Bazzocchi, M., \& Di Prampero, P. E. (1996). Primary motor and sensory cortex activation during motor performance and motor imagery: A functional magnetic resonance imaging study. Journal of Neuroscience, 16, 7688-7698. 
Presson, C. C. (1982). Strategies in spatial reasoning. Journal of Experimental Psychology: Learning, Memory, \& Cognition, 8, 243-251.

Raichle, M. E., MacLeod, A. M., Snyder, A. Z, Powers, W. J., GusNARD, D. A., \& Shulman, G. L. (2001). A default mode of brain function. Proceedings of the National Academy of Sciences, 98, 676-682.

Rao, S. M., Binder, J. R., Bandettini, P. A., Bobholz, J. A., Frost, J. A., My Klebust, B. M., Jacobson, R. D., \& Hyde, J. S. (1995). Somatotopic mapping of the human primary motor cortex with functional magnetic resonance imaging. Neurology, 45, 919-924.

Richter, W., Somorjai, R., Summers, R, Jarmasz, M., Menon, R. S., Gati, J. S., Georgopolous, A. P., Tegeler, C., Ugurbil, K., \& Kim, S. (2000). Motor area activity during mental rotation studies by timeresolved single-trial fMRI. Journal of Cognitive Neuroscience, 12, 310-320.

Shepard, R. N., \& Cooper, L. A. (1982). Mental images and their transformations. Cambridge, MA: MIT Press.

Shepard, R. N., \& Metzler, J. (1971). Mental rotation of threedimensional objects. Science, 171, 701-703.

Shulman, G. L., Fiez, J. A., Corbetta, M., Buckner, R. L., Miezin, F. M., Raichle, M. E., \& Peterson, S. E. (1997). Common blood flow changes across visual tasks: II. Decreases in cerebral cortex. Journal of Cognitive Neuroscience, 9, 648-663.

Stephan, K. M., Fink, G. R. Passingham, R. E., Silbersweig, D., Ceballos-Baumann, A. O., Frith, C. D., \& Frackowiak, R. S. J. (1995). Functional anatomy of the mental representation of upper extremity movements in healthy subjects. Journal of Neurophysiology, 73, 373-386.

Tagaris, G. A., Kim, S., Strupp, J. P., Anderson, P., Ugurbil, K., \& GEORGOPOULOS, A. P. (1996). Quantitative relations between parietal activation and performance in mental rotation. NeuroReport, 7, 773-776.

Tagaris, G. A., Kim, S., Strupp, J. P., Anderson, P., Ugurbil, K., \& Georgopolous, A. P. (1997). Mental rotation studies by functional magnetic resonance imaging at high field (4Tesla): Performance and cortical activation. Journal of Cognitive Neuroscience, 9, 419-432.

TAlairach, J., \& Tournoux, P. (1988). Co-planar stereotaxic atlas of the human brain. New York: Thieme Medical.

Tootell, R. B., Reppas, J. B., Kwong, K. K., Malach, R., Born, R. T., \& BRADY, T. J. (1995). Functional analysis of human MT and related visual cortical areas using magnetic resonance imaging. Journal of Neuroscience, 15, 3215-3230.

Vallar, G., Lobel, E., Galati, G., Berthoz, A., Pizzamiglio, L., \& LE BIHAN, D. (1999). A fronto-parietal system for computing the ego- centric spatial frame of reference in humans. Experimental Brain Research, 124, 281-286.

VindRas, P., \& ViViani, P. (1998). Frames of reference and control parameters in visuomanual pointing. Journal of Experimental Psychology: Human Perception \& Performance, 24, 569-591.

Wexler, M., Kosslyn, S. M., \& Berthoz, A. (1998). Motor processes in mental rotation. Cognition, $\mathbf{6 8}, 77-94$.

WohlschläGER, A., \& WohlschläGER, A. (1998). Mental and manual rotation. Journal of Experimental Psychology: Human Perception \& Performance, 24, 397-412.

Wraga, M., Creem, S. H., \& Proffitt, D. R. (2000). Updating displays after imagined object and viewer rotations. Journal of Experimental Psychology: Learning, Memory, \& Cognition, 26, 151-168.

Zacks, J., Rypma, B., Gabrieli, J. D. E., Tversky, B., \& Glover, G. H (1999). Imagined transformations of bodies: An fMRI investigation. Neuropsychologia, 37, 1029-1040.

\section{NOTES}

1. Volumes varied as a function of how quickly subjects performed the task.

2. TR corresponds to volume of 26 slices.

3 . Voxels that did not exceed the threshold were averaged in as zeros.

4. A similar analysis compared the fMRI data of the 6 subjects for whom behavioral data were analyzed with that of the 6 subjects lacking behavioral data (see Note 5).

5. Data from the first 6 subjects were not included in the analysis because of an error in the program recording the reaction time data.

6 . Two subjects were removed for extremely long RTs ( $>10 \mathrm{sec}$ on $50 \%$ of the trials), and 2 subjects were removed for being less than $50 \%$ accurate.

7. Mean correlation values $(p<.005)$ for 6 subjects with and without behavioral data also indicated similar significant superior parietal activity.

8. Parsons et al. (1995) also found another more lateral and anterior parietal region bilaterally in their hand-judgment task that was not found in the present data $(x, y, z:-30,-60,50 ; 34,-54,48)$.

9. The program AlphaSim estimated that a cluster size $>140 \mathrm{~mm}^{3}$ was statistically significant $(\alpha=.05)$ for a threshold of $p<.005$.

(Manuscript received January 4, 2001; revision accepted for publication June 14, 2001.) 